\title{
Mortality in British vegetarians
}

\author{
Paul N Appleby ${ }^{1, *}$, Timothy J Key ${ }^{1}$, Margaret Thorogood ${ }^{2}$, Michael L Burr ${ }^{3}$ and Jim Mann ${ }^{4}$ \\ ${ }^{1}$ Imperial Cancer Research Fund, Cancer Epidemiology Unit, Gibson Building, The Radcliffe Infirmary, Oxford \\ OX2 6HE, UK: ${ }^{2}$ Department of Public Health and Policy, London School of Hygiene and Tropical Medicine, \\ London, UK: ${ }^{3}$ Centre for Applied Public Health Medicine, University of Wales College of Medicine, Cardiff, UK: \\ ${ }^{4}$ Department of Human Nutrition, University of Otago, Dunedin, New Zealand
}

\section{Submitted 20 April 2001: Accepted 11 July 2001}

\begin{abstract}
Objective: To compare the mortality of British vegetarians and non-vegetarians. Design: Analysis of original data from two prospective studies each including a large proportion of vegetarians - the Oxford Vegetarian Study and the Health Food Shoppers Study. Standardised mortality ratios (SMRs) compared with the population of England and Wales were calculated from deaths before age 90 for vegetarians and nonvegetarians in each study. Death rate ratios (DRRs) for vegetarians compared with nonvegetarians within each study were calculated for each of 14 major causes of death.

Setting: UK.

Subjects: Twenty-one thousand men and women aged 16-89 years at recruitment, including more than 8000 vegetarians.

Results: SMRs for all causes of death were significantly below the reference level of 100 in both studies: 52 (95\% confidence interval (CI) 49-56) based on 1131 deaths in the Oxford Vegetarian Study and 59 (57-61) based on 2346 deaths in the Health Food Shoppers Study. For all causes of death, the DRR for vegetarians compared with nonvegetarians was close to one in both studies: 1.01 (95\% CI 0.89-1.14) in the Oxford Vegetarian Study, 1.03 (0.95-1.13) in the Health Food Shoppers Study.

Conclusions: British vegetarians have low mortality compared with the general population. Their death rates are similar to those of comparable non-vegetarians, suggesting that much of this benefit may be attributed to non-dietary lifestyle factors such as a low prevalence of smoking and a generally high socio-economic status, or to aspects of the diet other than the avoidance of meat and fish.
\end{abstract}

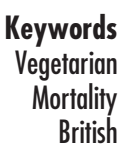

Vegetarian and semi-vegetarian diets have become increasingly popular in Western countries in recent years, although vegetarians still comprise only a small percentage of the population. There is evidence to suggest that vegetarian diets confer some health benefits, although concerns have also been expressed over possible nutritional deficiencies ${ }^{1-4}$.

The present analysis examines mortality in two prospective studies - the Oxford Vegetarian Study and the Health Food Shoppers Study. Each study recruited about 11000 health-conscious subjects living in the UK, including more than 8000 vegetarians overall. Some mortality data from these studies have already been published $^{5-9}$. These studies have also contributed data to a collaborative analysis of mortality in Western vegetarians that also included data from two studies of Californian Seventh-day Adventists (the Adventist Mortality Study and the Adventist Health Study) and the Heidelberg Study in Germany $^{10,11}$. This collaborative analysis showed that vegetarians have lower mortality from ischaemic heart disease than comparable non-vegetarians, but found no significant differences in mortality from other major causes of death including cerebrovascular disease and cancers of the stomach, colon and rectum, lung, breast and prostate, or for all-cause mortality.

The aim of this paper is to compare mortality for vegetarians with non-vegetarians in Britain after an extended period of follow-up involving nearly 3500 deaths in 400000 person-years of observation. We first describe standardised mortality ratios (SMRs) for vegetarians and non-vegetarians in each study to show how mortality in these subjects compares with the general population, and then present death rate ratios (DRRs) comparing mortality in vegetarians with non-vegetarians. Results are presented separately for the two studies, but inferences are drawn from the overall picture.

\section{Subjects and methods}

Recruitment of subjects to the two studies considered here has been described previously ${ }^{5,9}$. Briefly, the Oxford Vegetarian Study cohort was recruited between 1980 and 
1984 through the Vegetarian Society of the UK and the news media, with non-vegetarians recruited by the vegetarian participants from among their friends and relatives. The Health Food Shoppers Study cohort was recruited between 1973 and 1979 from customers of health food shops, members of vegetarian societies and readers of relevant magazines. In both studies follow-up was by record linkage with the National Health Service Central Register.

Subjects in the Oxford Vegetarian Study were included in the analysis if they were aged 16-89 years at entry, had not been diagnosed with a malignant cancer before recruitment (except for non-melanoma skin cancer, ICD9 code 173), and could be classified according to their diet and smoking habits. Subjects in the study were also classified according to their social class, body mass index, and whether or not they had pre-existing disease at recruitment, this being defined as previous or current diabetes, hypertension, angina, heart disease or stroke. Similarly, subjects in the Health Food Shoppers Study were included in the analysis if they were aged 16-89 years at entry, had not been diagnosed with a malignant cancer before recruitment (excluding non-melanoma skin cancer), and could be classified according to their diet and smoking habits. Data on social class, body mass index and pre-existing disease were not available in this study. Eight hundred and fifty subjects were common to both studies. These subjects contributed person-years to the Health Food Shoppers Study analysis up to their date of recruitment to the Oxford Vegetarian Study, and to the Oxford Vegetarian Study analysis thereafter.

Consistency of dietary practice was estimated by comparing the diets of the subjects common to both studies, and of nearly 3000 subjects in the Oxford Vegetarian Study who were also recruited into the Oxford arm of the European Prospective Investigation into Cancer and Nutrition (EPIC) Study ${ }^{12}$. Most of these subjects were recruited into the EPIC Study in 1994, at least 10 years after joining the Oxford Vegetarian Study. As subjects in the EPIC Study were also asked whether or not they ate meat or fish, it was possible to cross-classify subjects in both studies by their vegetarian status.

Subject to censoring at age 90, subjects were followed up to 30 June 2000 in the Oxford Vegetarian Study and 31 December 1997 in the Health Food Shoppers Study (active follow-up of the latter study was discontinued in 1999). Those who died were coded for underlying cause of death using the ninth revision of the International Classification of Diseases (ICD9). Standardised mortality ratios (SMRs) were calculated by comparison with mortality data for England and Wales for the period 1970-1998 using the Person-Years computer program ${ }^{13}$. Death rate ratios (DRRs) for vegetarians compared with non-vegetarians were calculated by Cox regression using the STATA statistical package ${ }^{14}$. In the Oxford Vegetarian Study, vegetarians were subjects who stated that they never ate meat or fish; in the Health Food Shoppers Study, vegetarians were subjects who answered yes to the question 'Are you a vegetarian?' but no data on either meat or fish consumption were available in this study. In order to compare results for the Oxford Vegetarian Study with those published in $1994^{5}$, we also calculated death rate ratios for non-meat eaters compared with meat eaters in this study.

All death rate ratios were adjusted for age at recruitment (in 11 categories: <40, 40-44, 45-49, 50-54, 55-59, 60$64,65-69,70-74,75-79,80-84,85-89$ years), sex and smoking habits (in three categories: non-smokers, light smokers and heavy smokers). Heavy smokers were subjects smoking 15 or more cigarettes per day, and light smokers were all other current smokers, including those smoking less than this amount or an unknown number of cigarettes per day, and other tobacco users. Non-smokers included former smokers as well as never smokers. Information on past smoking habits was not available in the Health Food Shoppers Study, and a preliminary analysis of data from the Oxford Vegetarian Study showed that adjusting for past smoking had very little effect on the death rate ratios. Similarly, adjusting the Oxford Vegetarian Study data for social class (also unavailable in the Health Food Shoppers Study) had almost no effect on the death rate ratios.

Death rate ratios were also calculated with the first five years of follow-up excluded in order to minimise the 'healthy volunteer effect'. We calculated death rate ratios in the Oxford Vegetarian Study further adjusted for preexisting disease to investigate the influence of this factor. In order to look for sex-specific differences, we also calculated death rate ratios for men and women separately in each study, adjusted for age and smoking. Mortality in middle age was examined by calculating death rate ratios in each study subject to censoring at age 70 , adjusted for age, sex and smoking.

Death rate ratios were calculated for each of the following causes of death: all causes (ICD9 codes 1-999); all malignant neoplasms (140-208), including colorectal cancer (153-154), lung cancer (162), female breast cancer (174) and prostate cancer (185); mental and neurological diseases (290-389); circulatory diseases (390-459), including ischaemic heart disease (410-414) and cerebrovascular disease (430-438); respiratory diseases (460-519); digestive diseases (520-579); injury and poisoning (800-999); and all other causes (001-139, 210-289, 580-799).

SMRs were calculated for a similar but slightly more detailed list of causes of death. In order to investigate variations in SMR with follow-up period, SMRs were also calculated by 5-year follow-up period for all causes of death, all malignant neoplasms and circulatory diseases.

\section{Results}

A total of 11045 Oxford Vegetarian Study subjects and 10736 Health Food Shoppers Study subjects were 
Table 1 Baseline characteristics of the subjects by study and diet, given as number (percentage) of subjects except where indicated. Subjects in both studies are counted in both halves of the table

\begin{tabular}{|c|c|c|c|c|}
\hline \multirow[b]{2}{*}{ Characteristic } & \multicolumn{2}{|c|}{$\begin{array}{l}\text { Oxford Vegetarian Study } \\
\qquad(n=11045)\end{array}$} & \multicolumn{2}{|c|}{$\begin{array}{l}\text { Health Food Shoppers Study } \\
\qquad(n=10736)\end{array}$} \\
\hline & Non-vegetarians & Vegetarians & Non-vegetarians & Vegetarians \\
\hline Number (\%) & $6371(57.7)$ & $4674(42.3)$ & $6136(57.2)$ & $4600(42.8)$ \\
\hline Female & $3800(59.6)$ & 3071 (65.7) & 3654 (59.6) & 2757 (59.9) \\
\hline Median age at entry (years) & 34 & 32 & 45 & 46 \\
\hline \multicolumn{5}{|l|}{ Smoking* } \\
\hline Non-smokers & 4917 (77.2) & 3980 (85.2) & 4719 (76.9) & 3953 (85.9) \\
\hline Light smokers & $864(13.6)$ & $452(9.7)$ & $902(14.7)$ & $458(10.0)$ \\
\hline Heavy smokers & $590(9.3)$ & $242(5.2)$ & $515(8.4)$ & $189(4.1)$ \\
\hline \multicolumn{5}{|l|}{ Social class } \\
\hline $\mathrm{I}-\mathrm{II}$ & 3197 (50.2) & $2094(44.8)$ & NA§ & NA \\
\hline III-V & $1862(29.2)$ & $1296(27.7)$ & NA & NA \\
\hline Unclassified & $1312(20.6)$ & $1284(26.5)$ & NA & NA \\
\hline \multicolumn{4}{|l|}{ Body mass index $\ddagger\left(\mathrm{kg} \mathrm{m}^{-2}\right)$} & NA \\
\hline$<20$ & $1065(17.0)$ & $1320(28.8)$ & NA & NA \\
\hline $20-22.5$ & 2480 (39.5) & 1906 (41.6) & NA & NA \\
\hline $22.5-25$ & $1714(27.3)$ & $964(21.0)$ & NA & NA \\
\hline$\geq 25$ & $1013(16.2)$ & $396(8.6)$ & NA & NA \\
\hline Median & 22.1 & 21.1 & NA & NA \\
\hline
\end{tabular}

included in the analysis. Forty-two per cent of Oxford Vegetarian Study subjects and 43\% of Health Food Shoppers Study subjects were vegetarians (Table 1). About $60 \%$ of subjects in both studies were female. Age at recruitment was similar among vegetarians and nonvegetarians in both studies, but Oxford Vegetarian Study subjects were generally considerably younger than Health Food Shoppers Study subjects (median ages at recruitment were 33 and 46 years, respectively). Mean follow-up was 17.6 years in the Oxford Vegetarian Study and 18.7 years in the Health Food Shoppers Study. There was a low incidence of smoking at about $20 \%$ in both studies, with a lower proportion of smokers among the vegetarians. Information on social class, body mass index and preexisting disease was only available in the Oxford Vegetarian Study. The incidence of pre-existing disease was similar for non-vegetarians and vegetarians (7.9\% vs. $7.3 \%$ ), but median body mass index was noticeably lower among the vegetarians $\left(21.1 \mathrm{~kg} \mathrm{~m}^{-2}\right.$ vs. $\left.22.1 \mathrm{~kg} \mathrm{~m}^{-2}\right)$. Forty-four per cent of the non-vegetarians in the Oxford Vegetarian Study reported eating meat on a daily basis, with an estimated weekly intake of $640 \mathrm{~g}$. Data on meat consumption were unavailable for the Health Food Shoppers Study.

Of 850 subjects common to both studies, 767 (90\%) were allocated to the same diet group (vegetarian or nonvegetarian) in both analyses. Similarly, of 2946 subjects in the Oxford Vegetarian Study who were later recruited into the Oxford arm of the EPIC study, 2571 (87\%) remained in the same diet group.
There were 1131 deaths before age 90 in the Oxford Vegetarian Study and 2346 deaths before age 90 in the Health Food Shoppers Study. Standardised mortality ratios for selected causes of death are shown by study and by diet in Table 2. SMRs for all causes of death combined were significantly lower than in the reference population in both studies. Expressed as a percentage, the values were 52\% (95\% confidence interval (CI) 49-56\%) in the Oxford Vegetarian Study and 59\% (57-61\%) in the Health Food Shoppers Study. All-cause SMRs were similar for vegetarians and non-vegetarians in both studies (51\% vs. $54 \%$ in the Oxford Vegetarian Study, both 59\% in the Health Food Shoppers Study). The SMRs for almost all of the causes of death tabulated were below 100\%, and many were significantly lower than 100\% (none were significantly higher than 100\%), reflecting the generally good level of health and low prevalence of smoking among the participants in both studies.

Figure 1 shows SMRs by study, diet and 5-year followup period for all malignant neoplasms, circulatory diseases and all causes of death. SMRs were similar in each diet group in each of the 5-year follow-up periods for the Health Food Shoppers Study. However, in the Oxford Vegetarian Study, the SMRs were noticeably lower among vegetarians than among non-vegetarians in the first five years of the study, but were similar thereafter. SMRs for vegetarians during the first five years of follow-up in this study were 25\% (95\% CI 14-42\%) for all malignant neoplasms, 31\% (21-43\%) for circulatory diseases and $35 \%(28-44 \%)$ for all causes of death. The corresponding 
Table 2 Number of deaths (D) and standardised mortality ratios expressed as a percentage (95\% confidence intervals) calculated from deaths before age 90 years for selected causes of death by study and diet

\begin{tabular}{|c|c|c|c|c|c|c|c|c|}
\hline \multirow[b]{3}{*}{ Cause of death (ICD9 codes) } & \multicolumn{4}{|c|}{ Oxford Vegetarian Study } & \multicolumn{4}{|c|}{ Health Food Shoppers Study } \\
\hline & \multicolumn{2}{|c|}{ Non-vegetarians } & \multicolumn{2}{|c|}{ Vegetarians } & \multicolumn{2}{|c|}{ Non-vegetarians } & \multicolumn{2}{|c|}{ Vegetarians } \\
\hline & $\mathrm{D}$ & SMR & $\mathrm{D}$ & SMR & $\mathrm{D}$ & SMR & $\mathrm{D}$ & SMR \\
\hline All causes of death $(1-999)$ & 599 & $54(49-58)$ & 532 & $51(47-56)$ & 1383 & $59(56-62)$ & 963 & $59(55-63)$ \\
\hline All malignant neoplasms (140-208) & 211 & $69(60-79)$ & 156 & $59(50-70)$ & 381 & $63(57-70)$ & 256 & $70(62-79)$ \\
\hline Stomach cancer (151) & 6 & $36(13-77)$ & 4 & $26(7-67)$ & 21 & $51(32-79)$ & 16 & $59(34-95)$ \\
\hline Colorectal cancer (153-154) & 24 & $70(45-104)$ & 25 & $82(53-122)$ & 61 & $85(65-109)$ & 33 & $70(48-98)$ \\
\hline Cancer of the pancreas (157) & 10 & $79(38-145)$ & 7 & $63(25-131)$ & 22 & $85(53-128)$ & 16 & $98(56-159)$ \\
\hline Lung cancer (162) & 27 & $38(25-55)$ & 16 & $27(15-44)$ & 53 & $35(26-45)$ & 24 & $27(17-40)$ \\
\hline Female breast cancer (174) & 24 & $69(44-103)$ & 22 & $74(46-111)$ & 43 & $72(52-97)$ & 41 & $112(80-151)$ \\
\hline Ovarian cancer (183) & 12 & $114(59-200)$ & 9 & $102(47-194)$ & 17 & $91(53-145)$ & 9 & $83(38-157)$ \\
\hline Prostate cancer (185) & 18 & $117(69-185)$ & 8 & $58(25-115)$ & 22 & $71(44-107)$ & 16 & $83(48-136)$ \\
\hline $\begin{array}{l}\text { Bladder and other urinary cancer } \\
(188-189, \text { excluding 189.0) }\end{array}$ & 3 & $31(6-91)$ & 5 & $58(19-135)$ & 10 & $48(23-89)$ & 9 & $68(31-129)$ \\
\hline Benign and unspecified neoplasms (210-239) & 4 & $101(28-259)$ & 1 & $29(1-161)$ & 2 & $28(3-103)$ & 2 & $46(6-165)$ \\
\hline Endocrine diseases $(240-279)$ & 3 & $17(4-51)$ & 8 & $51(22-101)$ & 19 & $56(34-88)$ & 12 & $53(27-92)$ \\
\hline Diabetes mellitus (250) & 1 & $7(0-42)$ & 4 & $32(9-83)$ & 8 & $30(13-59)$ & 4 & $22(6-57)$ \\
\hline Diseases of the blood (280-289) & 1 & $26(1-143)$ & 2 & $52(6-189)$ & 2 & $25(3-92)$ & 3 & $52(11-152)$ \\
\hline Mental disorders $(290-319)$ & 4 & $23(6-58)$ & 11 & $61(31-109)$ & 9 & $28(13-54)$ & 9 & $38(17-72)$ \\
\hline Diseases of the nervous system $(320-389)$ & 8 & $39(17-77)$ & 13 & $70(37-120)$ & 27 & $74(49-107)$ & 21 & $87(54-133)$ \\
\hline Circulatory diseases $(390-459)$ & 255 & $52(46-59)$ & 214 & $45(40-52)$ & 672 & $60(56-65)$ & 445 & $55(50-60)$ \\
\hline Ischaemic heart disease $(410-414)$ & 141 & $50(42-59)$ & 109 & $42(34-51)$ & 359 & $57(51-63)$ & 203 & $47(41-54)$ \\
\hline Cerebrovascular disease $(430-438)$ & 62 & $52(40-66)$ & 63 & $52(40-67)$ & 189 & $69(59-79)$ & 141 & $66(56-78)$ \\
\hline Respiratory diseases $(460-519)$ & 46 & $33(24-44)$ & 61 & $44(33-56)$ & 146 & $48(40-56)$ & 119 & $51(42-61)$ \\
\hline Digestive diseases $(520-579)$ & 20 & $52(31-80)$ & 20 & $57(35-88)$ & 32 & $44(30-63)$ & 20 & $40(25-62)$ \\
\hline Genitourinary diseases (580-629) & 9 & $68(31-129)$ & 8 & $59(25-116)$ & 20 & $66(40-101)$ & 15 & $65(36-107)$ \\
\hline Injury and poisoning (800-999) & 27 & $74(49-107)$ & 21 & $71(44-109)$ & 47 & $85(63-113)$ & 42 & $109(78-147)$ \\
\hline All other causes $(001-139,630-799)$ & 11 & $40(20-72)$ & 17 & $65(38-104)$ & 26 & $57(37-83)$ & 19 & $56(34-87)$ \\
\hline
\end{tabular}

values for non-vegetarians were $74 \%$ (55-98\%), 49\% (3664\%) and 54\% (45-64\%), respectively.

The death rate ratios for vegetarians compared with non-vegetarians in each of 14 causes of death categories, adjusted for age, sex and smoking, are shown in Table 3. For all causes of death, the DRR was close to one in both studies: 1.01 (95\% CI 0.89-1.14) in the Oxford Vegetarian Study, $1.03(0.95-1.13)$ in the Health Food Shoppers Study. For two causes of death the DRR was significantly greater than one: female breast cancer in the Health Food Shoppers Study (1.73 (1.11-2.69), $P=0.015)$ and mental and neurological diseases in the Oxford Vegetarian Study (2.46 (1.21-5.01), $P=0.013)$. The corresponding DRR in the other study for these causes of death was not statistically significant, although each value was greater than one. No DRRs were significantly less than one for any cause of death in either study, although the DRR for ischaemic heart disease in the Health Food Shoppers Study was of borderline significance $(0.85$ (0.71-1.01), $P=0.070$ ).

Death rate ratios for vegetarians compared with nonvegetarians for men and women separately were broadly similar to those for both sexes combined (results not shown). When we compared mortality up to age 70 years, the DRRs for all causes of death were 0.95 (0.76-1.19), based on 333 deaths, and 1.13 (0.95-1.33), based on 637 deaths, in the Oxford Vegetarian and Health Food Shoppers studies, respectively. The DRRs for female breast cancer in the Health Food Shoppers Study and for mental and neurological diseases in the Oxford Vegetarian Study were both significantly greater than one: 2.02 (1.18$3.47), P=0.011$ and $4.25(1.26-14.3), P=0.020$, respectively. Conversely, the DRR for circulatory diseases was significantly less than one in the Oxford Vegetarian Study (0.62 (0.39-0.99), $P=0.047)$. No other DRRs were statistically significant.

When the first five years of follow-up were excluded, the DRRs showed little change in the Health Food Shoppers Study but generally increased in the Oxford Vegetarian Study (Table 4). For all causes of death, the DRR increased to $1.09(0.96-1.25)$ in the Oxford Vegetarian Study and was virtually unchanged at 1.05 (0.96-1.15) in the Health Food Shoppers Study. The magnitude of the DRR for the two causes of death giving a significant result in Table 3 was essentially unchanged, and both remained statistically significant at the 5\% level. Again, no DRRs were significantly less than one for any cause of death in either study.

We investigated the effect of further adjusting for preexisting disease in the Oxford Vegetarian Study. Most DRRs increased slightly, as might be expected given the lower prevalence of pre-existing disease among the vegetarians. For example, the DRRs for all causes of death, all malignant neoplasms and circulatory diseases increased to $1.03(0.91-1.16), 0.90(0.73-1.11)$ and 0.97 (0.80-1.17), respectively, representing increases of $2 \%$, 
Oxford Vegetarian Study
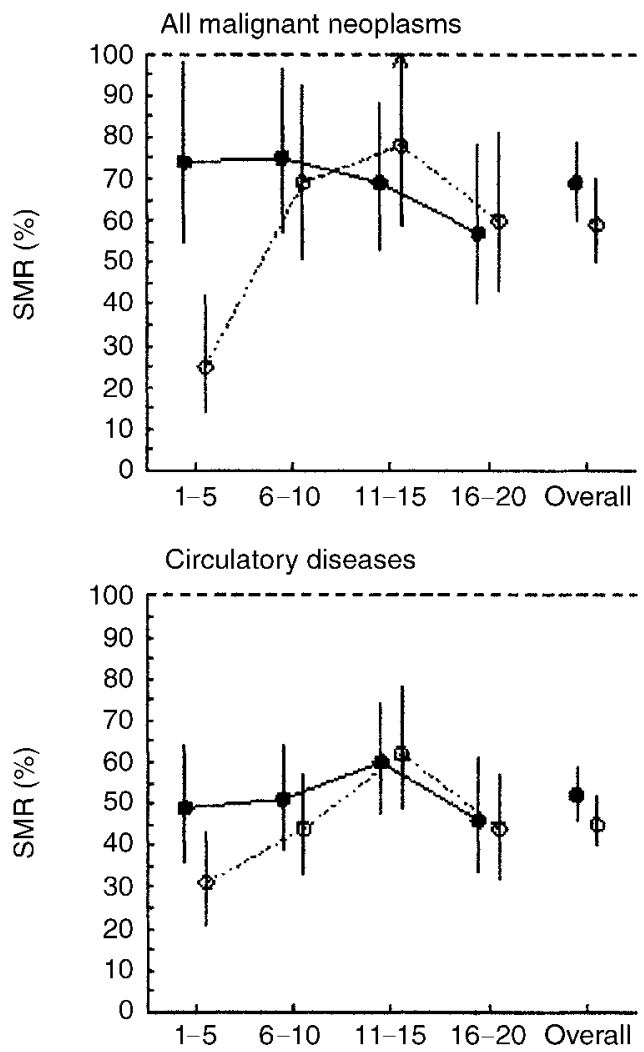

All causes of death

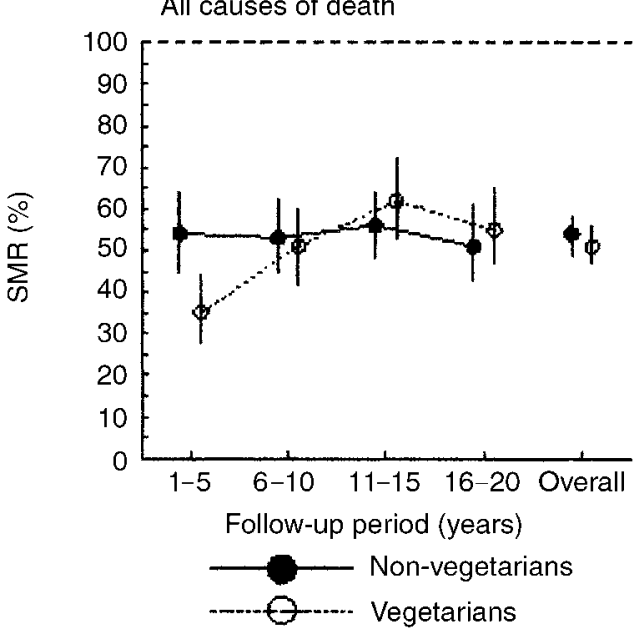

Health Food Shoppers Study

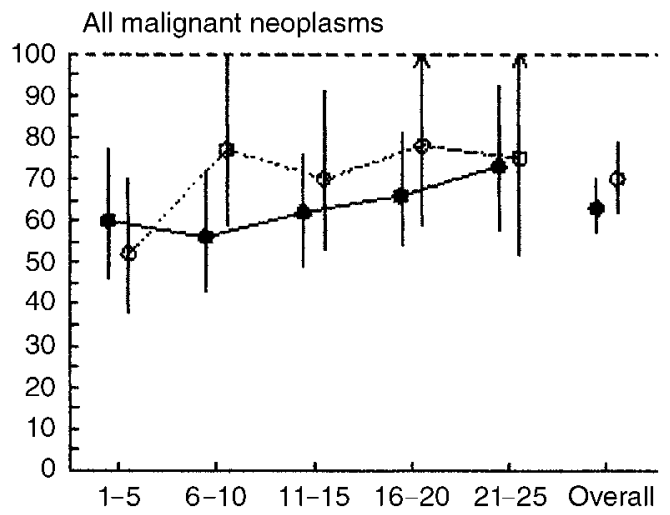

Circulatory diseases

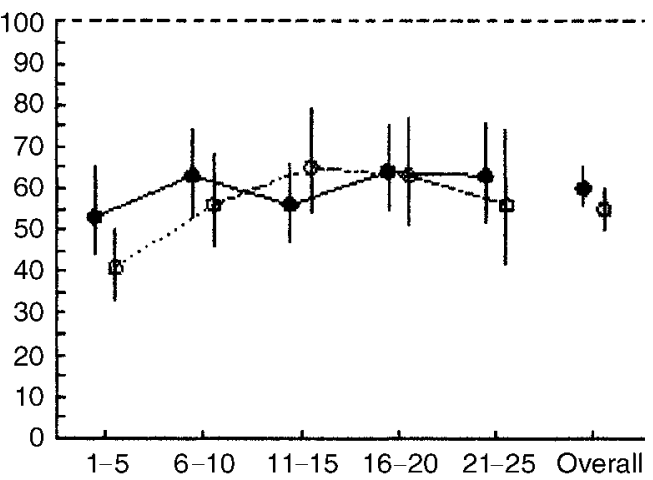

All causes of death

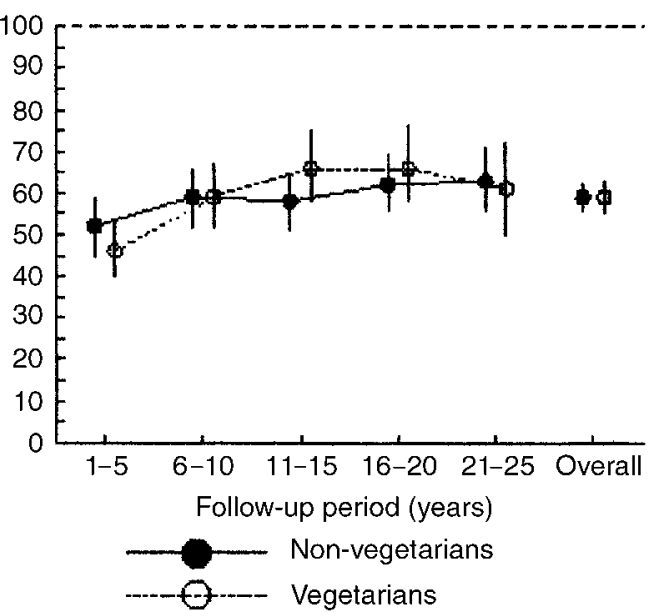

Fig. 1 Standardised mortality ratios (SMRs) and their 95\% confidence intervals, expressed as a percentage, for all malignant neoplasms (ICD9 140-208), circulatory diseases (ICD9 390-459) and all causes of death, by study, diet and 5-year follow-up period. SMRs are represented by black circles joined by solid lines for non-vegetarians and by white circles joined by dotted lines for vegetarians. A solid vertical line through each circle represents the 95\% confidence interval. Overall SMRs and their 95\% confidence intervals for each diet group are shown on the right-hand side of each plot

$1 \%$ and $4 \%$ respectively over the corresponding values in Table 3. The DRR for mental and neurological diseases (2.50 (1.23-5.09), $P=0.012$ ) remained the only one to reach statistical significance.

In order to facilitate comparison with an earlier publication $^{5}$, we calculated DRRs for non-meat eaters compared with meat eaters in the Oxford Vegetarian
Study, adjusted for age, sex and smoking, both with and without data from the first five years of follow-up. The 6060 non-meat eaters included 1386 'semi-vegetarians' who either ate meat less than once per week on average, or ate no meat but did eat fish, whereas the 4985 meat eaters all ate meat at least once per week. DRRs were generally lower than the corresponding values for 
Table 3 Number of deaths and death rate ratios (95\% confidence intervals) for vegetarians (V) versus non-vegetarians (NV) by study, adjusted for age at entry, sex and smoking

\begin{tabular}{|c|c|c|c|c|c|c|}
\hline \multirow[b]{2}{*}{ Cause of death (ICD9 codes) } & \multicolumn{3}{|c|}{ Oxford Vegetarian Study } & \multicolumn{3}{|c|}{ Health Food Shoppers Study } \\
\hline & NV & V & DRR $(95 \% \mathrm{Cl})$ & NV & V & DRR $(95 \% \mathrm{Cl})$ \\
\hline All causes of death (1-999) & 599 & 532 & $1.01(0.89-1.14)$ & 1383 & 963 & $1.03(0.95-1.13)$ \\
\hline All malignant neoplasms (140-208) & 211 & 156 & $0.89(0.72-1.10)$ & 381 & 256 & $1.12(0.95-1.32)$ \\
\hline Colorectal cancer (153-154) & 24 & 25 & $1.20(0.68-2.13)$ & 61 & 33 & $0.79(0.51-1.22)$ \\
\hline Lung cancer (162) & 27 & 16 & $0.82(0.44-1.56)$ & 53 & 24 & $1.05(0.64-1.72)$ \\
\hline Female breast cancer (174) & 24 & 22 & $1.02(0.57-1.84)$ & 43 & 41 & $1.73(1.11-2.69)$ \\
\hline Prostate cancer (185) & 18 & 8 & $0.50(0.22-1.17)$ & 22 & 16 & $1.24(0.64-2.41)$ \\
\hline Mental and neurological diseases (290-389) & 12 & 24 & $2.46(1.21-5.01)$ & 36 & 30 & $1.36(0.83-2.23)$ \\
\hline Circulatory diseases (390-459) & 255 & 214 & $0.93(0.77-1.12)$ & 672 & 445 & $0.95(0.84-1.07)$ \\
\hline Ischaemic heart disease (410-414) & 141 & 109 & $0.86(0.67-1.12)$ & 359 & 203 & $0.85(0.71-1.01)$ \\
\hline Cerebrovascular disease (430-438) & 62 & 63 & $1.08(0.75-1.54)$ & 189 & 141 & $0.99(0.79-1.24)$ \\
\hline Respiratory diseases (460-519) & 46 & 61 & $1.42(0.96-2.10)$ & 146 & 119 & $1.07(0.84-1.38)$ \\
\hline Digestive diseases (520-579) & 20 & 20 & $1.13(0.59-2.15)$ & 32 & 20 & $0.79(0.44-1.44)$ \\
\hline Injury and poisoning (800-999) & 27 & 21 & $1.01(0.56-1.81)$ & 47 & 42 & $1.34(0.87-2.06)$ \\
\hline All other causes $(001-139,210-289,580-799)$ & 28 & 36 & $1.20(0.73-2.00)$ & 69 & 51 & $1.06(0.73-1.53)$ \\
\hline
\end{tabular}

vegetarians compared with non-vegetarians in Tables 3 and 4. In particular, the DRRs for all causes of death were 0.96 (0.85-1.08) for the complete follow-up period, and 1.05 (0.92-1.21) when the first five years of follow-up were excluded. The DRR was significantly less than one for prostate cancer $(0.43(0.19-0.97), P=0.043)$, greater than one for mental and neurological diseases (2.42 (1.115.28), $P=0.026)$, and marginally significant for ischaemic heart disease $(0.77(0.60-1.00), P=0.052)$. However, no DRRs were statistically significant when the first five years of follow-up were excluded.

\section{Discussion}

The results presented in this paper summarise relative mortality for vegetarians and non-vegetarians among about 21000 health-conscious Britons. Data for 850 subjects common to both studies, and for nearly 3000 subjects in the Oxford Vegetarian Study who were recruited into the Oxford arm of the EPIC Study at least
10 years later, suggest a high level of dietary consistency. Both vegetarians and non-vegetarians enjoy low mortality compared with the general population, with an overall standardised mortality ratio of $50-60 \%$. It is clear from this that both groups are atypical of the general population. For example, the non-vegetarians in the Oxford Vegetarian Study eat considerably less meat (mean $640 \mathrm{~g}$ per week) than the average of $1050 \mathrm{~g}$ per week reported in the Dietary and Nutritional Survey of British Adults of 1986/ $87^{15}$. The low prevalence of smoking and generally high socio-economic status of the participants, as well as other lifestyle and dietary factors, added to the 'healthy volunteer effect', probably accounts for this low mortality. Overall mortality is similar in both vegetarians and nonvegetarians, with death rate ratios near to one for all causes of death.

A previous analysis of data from the Oxford Vegetarian Study after 12 years follow-up showed lower mortality in non-meat eaters compared with meat eaters for all causes of death, ischaemic heart disease and all malignant

Table 4 Number of deaths and death rate ratios (95\% confidence intervals) for vegetarians (V) versus non-vegetarians (NV) by study, adjusted for age at entry, sex and smoking, excluding the first five years of follow-up

\begin{tabular}{|c|c|c|c|c|c|c|}
\hline \multirow[b]{2}{*}{ Cause of death (ICD9 codes) } & \multicolumn{3}{|c|}{ Oxford Vegetarian Study } & \multicolumn{3}{|c|}{ Health Food Shoppers Study } \\
\hline & NV & V & $\operatorname{DRR}(95 \% \mathrm{Cl})$ & NV & V & DRR $(95 \% \mathrm{Cl})$ \\
\hline All causes of death $(1-999)$ & 471 & 450 & $1.09(0.96-1.25)$ & 1176 & 769 & $1.05(0.96-1.15)$ \\
\hline All malignant neoplasms (140-208) & 162 & 141 & $1.06(0.85-1.34)$ & 321 & 210 & $1.18(0.99-1.41)$ \\
\hline Colorectal cancer (153-154) & 18 & 23 & $1.42(0.76-2.67)$ & 48 & 26 & $0.85(0.52-1.39)$ \\
\hline Lung cancer (162) & 16 & 14 & $1.27(0.60-2.68)$ & 44 & 21 & $1.19(0.70-2.02)$ \\
\hline Female breast cancer (174) & 19 & 21 & $1.23(0.66-2.31)$ & 39 & 34 & $1.62(1.01-2.60)$ \\
\hline Prostate cancer (185) & 14 & 8 & $0.66(0.27-1.59)$ & 18 & 13 & $1.29(0.62-2.68)$ \\
\hline Mental and neurological diseases (290-389) & 10 & 20 & $2.21(1.02-4.78)$ & 35 & 26 & $1.24(0.74-2.08)$ \\
\hline Circulatory diseases (390-459) & 202 & 180 & $1.01(0.82-1.24)$ & 566 & 355 & $0.97(0.85-1.12)$ \\
\hline Ischaemic heart disease (410-414) & 107 & 90 & $0.98(0.73-1.30)$ & 295 & 162 & $0.89(0.73-1.09)$ \\
\hline Cerebrovascular disease $(430-438)$ & 53 & 55 & $1.10(0.75-1.63)$ & 165 & 110 & $0.97(0.75-1.24)$ \\
\hline Respiratory diseases $(460-519)$ & 39 & 53 & $1.43(0.94-2.18)$ & 130 & 93 & $1.03(0.78-1.35)$ \\
\hline Digestive diseases $(520-579)$ & 17 & 12 & $0.87(0.41-1.85)$ & 26 & 17 & $0.93(0.49-1.77)$ \\
\hline Injury and poisoning (800-999) & 18 & 15 & $0.97(0.48-1.97)$ & 36 & 29 & $1.31(0.80-2.16)$ \\
\hline All other causes $(001-139,210-289,580-799)$ & 23 & 29 & $1.17(0.67-2.04)$ & 62 & 39 & $0.96(0.64-1.45)$ \\
\hline
\end{tabular}


neoplasms ${ }^{5}$. In the previous analysis, subjects who ate fish but not meat, or those who ate meat less than once per week, were included in the non-meat eaters, whereas, in the present analysis, these subjects were classified as nonvegetarians. Although more than $20 \%$ of the nonvegetarians belong to these 'semi-vegetarian' categories, we found only slight differences between the death rate ratios comparing non-meat eaters with meat eaters and those comparing vegetarians with non-vegetarians in this study.

At first sight the results for relative mortality reported here may seem surprising when compared with the earlier publication. In particular, the lower death rates for all malignant neoplasms, ischaemic heart disease and all causes of death among non-meat eaters have effectively disappeared. The main reason for this can be seen from an examination of Fig. 1. The substantially lower SMRs among vegetarians during the first five years of follow-up clearly have more effect on a mortality analysis performed after 12 years of follow-up than on one performed after 18 years of follow-up. A commentary on the earlier paper suggested that the results might have been due to selfselection by healthy vegetarians, resulting in a stronger 'healthy volunteer effect' among the non-meat eaters ${ }^{16}$. The Oxford Vegetarian Study had the acknowledged aim of recruiting vegetarians to study their health. It may be that vegetarians were reluctant to volunteer if they had an illness. Conversely, the non-vegetarian participants, who were recruited by the vegetarians, may have been less inclined to rule themselves out of the study on health grounds. In contrast, the Health Food Shoppers Study, which did not set out to recruit vegetarians per se, shows similar SMRs for vegetarians and non-vegetarians during the first five years of follow-up.

The lack of an association between vegetarian status and all-cause mortality contrasts with findings for the Adventist Mortality Study and the Adventist Health Study, which both showed a significantly lower mortality for vegetarians (by about 20\%) in the collaborative analysis ${ }^{11}$. Why this should be the case is unclear, although it has been noted that differences in disease rates between vegetarian and non-vegetarian Seventh-day Adventists cannot be ascribed only to the absence of meat ${ }^{17}$. In particular, vegetarian Seventh-day Adventists eat significantly more fruit, nuts and legumes, and significantly fewer eggs, than their non-vegetarian colleagues ${ }^{17}$, and are also less likely to smoke cigarettes or drink alcohol ${ }^{11}$. However, similar contrasts between diet groups are evident in the Oxford Vegetarian Study, with vegetarians and especially vegans, who consume no animal products, consuming more carbohydrate and dietary fibre and less saturated fat than non-vegetarians ${ }^{18}$. Therefore, other, non-dietary factors may account for the differences in relative mortality.

The death rate ratios for ischaemic heart disease reported here (0.86 (95\% CI 0.67-1.12) in the Oxford
Vegetarian Study and $0.85(0.71-1.01)$ in the Health Food Shoppers Study) are compatible with a moderate reduction in ischaemic heart disease mortality among vegetarians. In the collaborative analysis of mortality in vegetarians, the Adventist Mortality Study, the Adventist Health Study and the Heidelberg Study each showed a somewhat greater reduction in ischaemic heart disease mortality among vegetarians than we observe in the two British cohorts ${ }^{11}$. However, it should be noted that many different dietary factors have been associated with ischaemic heart disease mortality. For example, a previous analysis of data from the Oxford Vegetarian Study showed that consumption of both cheese and eggs (foods eaten by most vegetarians) were more important predictors of ischaemic heart disease mortality than either meat or fish ${ }^{6}$.

Two causes of death gave rise to a significantly higher mortality among vegetarians in one study but not in the other - female breast cancer in the Health Food Shoppers Study, and mental and neurological diseases in the Oxford Vegetarian Study. The higher mortality from breast cancer among vegetarian women in the Health Food Shoppers Study has been noted previously, and it was thought that this might partly reflect different reproductive patterns between the diet groups ${ }^{9}$. Data on parity were not available in this study, but in the Oxford Vegetarian Study vegetarian women had fewer live births on average than nonvegetarian women (mean 0.8 vs. 1.1), a difference which would be expected to increase their risk for breast cancer ${ }^{19}$. Despite this, there was no excess in breast cancer mortality among the vegetarian women in the Oxford Vegetarian Study. In the collaborative analysis, breast cancer mortality was significantly lower among vegetarians in the Adventist Health Study, with no significant difference overall ${ }^{11}$. Therefore, it seems likely that a vegetarian diet per se has little or no effect on breast cancer mortality.

We found a significantly higher mortality from mental and neurological diseases (ICD9 290-389) for vegetarians than for non-vegetarians in the Oxford Vegetarian Study. Mortality from this cause was also higher for vegetarians in the Health Food Shoppers Study, although the DRR was not statistically significant. This finding was not anticipated, and it should be noted that there were only 36 deaths from this end point in the Oxford Vegetarian Study. Mortality from mental and neurological diseases was not included in the collaborative analysis, although some evidence has emerged from sub-studies of participants in the Adventist Health Study. Giem and colleagues found a positive association between meat eating and dementia in a matched analysis of 272 subjects, but there was no significant difference in the incidence of dementia between vegetarians and meat eaters in a larger group of nearly 3000 unmatched subjects from this study ${ }^{20}$. In a separate study of 90 elderly members of the cohort there was no association between vegetarian status and cognitive function $^{21}$. Nevertheless, we believe that our finding merits further investigation. 
In conclusion, British vegetarians have low mortality compared with the general population. Much of this benefit may be attributed to non-dietary lifestyle factors such as a low prevalence of smoking and a generally high socio-economic status, or to aspects of the diet other than the avoidance of meat and fish; for example, a higher than average intake of vegetables and fruit. Non-vegetarians who share these characteristics also have low relative mortality, and there is little difference in overall mortality between vegetarians and comparable non-vegetarians. Mortality was not significantly lower among vegetarians for any of the causes of death studied, although results were consistent with a moderate reduction in mortality from ischaemic heart disease. Findings of higher mortality among vegetarians for breast cancer in women and for mental and neurological diseases reported here are not consistent with other studies of Western vegetarians, but merit further investigation. Data from the 58000 subjects in the Oxford arm of the European Prospective Investigation into Cancer and Nutrition (EPIC) Study ${ }^{12}$, 30\% of whom are vegetarians, should enable us to do this.

\section{Acknowledgements}

We thank all the participants in the Oxford Vegetarian Study and the Health Food Shoppers Study and all the scientists and clerical staff who have worked on these studies. This analysis was supported by the Imperial Cancer Research Fund.

\section{References}

1 Dwyer JT. Health aspects of vegetarian diets. Am. J. Clin. Nutr. 1988; 48: 712S-38S.

2 Thorogood M. The epidemiology of vegetarianism and health. Nutr. Res. Rev. 1995; 8: 179-92.

3 Sanders TAB. The nutritional adequacy of plant-based diets. Proc. Nutr. Soc. 1999; 58: 265-9.

4 Key TJ, Davey GK, Appleby PN. Health benefits of a vegetarian diet. Proc. Nutr. Soc. 1999; 58: 271-5.

5 Thorogood M, Mann J, Appleby P, McPherson K. Risk of death from cancer and ischaemic heart disease in meat and non-meat eaters. Br. Med. J. 1994; 308: 1667-70.
6 Mann JI, Appleby PN, Key TJ, Thorogood M. Dietary determinants of ischaemic heart disease in health conscious individuals. Heart 1997; 78: 450-5.

7 Burr ML, Sweetnam PM. Vegetarianism, dietary fibre, and mortality. Am. J. Clin. Nutr. 1982; 36: 873-7.

8 Burr ML, Butland BK. Heart disease in British vegetarians. Am. J. Clin. Nutr. 1988; 48: 830-2.

9 Key TJ, Thorogood M, Appleby PN, Burr ML. Dietary habits and mortality in 11000 vegetarians and health conscious people: results of a 17 year follow up. Br. Med.J. 1996; 313 : $775-9$.

10 Key TJ, Fraser GE, Thorogood M, Appleby PN, Beral V, Reeves G, Burr ML, Chang-Claude J, Frentzel-Beyme R, Kuzma JW, Mann J, McPherson K. Mortality in vegetarians and non-vegetarians: a collaborative analysis of 8300 deaths among 76000 men and women in five prospective studies. Public Health Nutr. 1998; 1: 33-41.

11 Key TJ, Fraser GE, Thorogood M, Appleby PN, Beral V, Reeves G, Burr ML, Chang-Claude J, Frentzel-Beyme R, Kuzma JW, Mann J, McPherson K. Mortality in vegetarians and non-vegetarians: detailed findings from a collaborative analysis of 5 prospective studies. Am. J. Clin. Nutr. 1999; 70(Suppl.): $516 \mathrm{~S}-24 \mathrm{~S}$.

12 Riboli E, Kaaks R. The EPIC Project: rationale and study design. Int. J. Epidemiol 1997; 26(Suppl.): 6S-14S.

13 Coleman MP, Hermon C, Douglas A. Person-years (PYRS): A Fortran Program for Cohort Study Analysis. IARC Internal Report No. 89. Lyon: World Health Organization, 1989.

14 Stata Corporation. Stata Statistical Software: Release 5.0. College Station, TX: Stata Press, 1997.

15 Gregory J, Foster K, Tyler H, Wiseman M. The Dietary and Nutritional Survey of British Adults. London: HMSO, 1990.

16 Vandenbroucke JP. Commentary: Should you eat meat, or are you confounded by methodological debate? $\mathrm{Br}$. Med. J. 1994; 308: 1671.

17 Fraser GE. Associations between diet and cancer, ischemic heart disease, and all-cause mortality in non-Hispanic white California Seventh-day Adventists. Am. J. Clin. Nutr. 1999; 70(Suppl.): 532S-8S

18 Thorogood M, Roe L, McPherson K, Mann J. Dietary intake and plasma lipid levels: lessons from a study of the diet of health conscious groups. Br. Med. J. 1990; 300: 1297-301.

19 Key TJ, Verkasalo PK, Banks E. Epidemiology of breast cancer. Lancet Oncol. 2001; 2: 133-40.

20 Giem P, Beeson WL, Fraser GE. The incidence of dementia and intake of animal products: preliminary findings from the Adventist Health Study. Neuroepidemiology 1993; 12: 28-36.

21 Fraser GE, Singh PN, Bennett H. Variables associated with cognitive function in elderly Californian Seventh-day Adventists. Am. J. Epidemiol. 1996; 143: 1181-90. 